VISUAL PSYCHOPHYSICS

\section{Illusory afterimages}

We are all familiar with afterimages. If you look at a bright light for a couple of seconds, then close your eyes, you will see a dark spot; similarly, gazing at a coloured shape can induce an afterimage in the complementary colour - for example, a red square will produce a green afterimage. This is usually attributed to bleaching of the pigments in the retinal photoreceptors or to neural adaptation in the retina. But Shimojo et al. have found evidence that some afterimages can result from adaptation at a cortical level.

They used a type of visual stimulus that is designed to cause 'filling-in', where the visual system draws on elements in a visual scene to 'complete' a shape that is not actually there. In the Varin configuration, for example, four circles like those depicted in the figure are arrayed in a square layout. In each circle, the quadrant (or wedge) nearest the centre of the square is coloured differently from the rest of the circles, so that the four coloured quadrants appear to form the corners of a smaller, coloured square seen through the circles or superimposed on them. Our visual system fills in the missing lines so that we perceive a complete square.

Looking at this stimulus for a while leads to the perception of an afterimage of a square, the colour of which is complementary to the perceived square's colour, as well as afterimages of the incomplete circles and the wedges. There are two possible mechanisms for the 'global afterimage' of the square. It could arise from the perceived afterimages of the circles and wedges (the 'local afterimages'), just as the original perception arises from the perception of the circles themselves; or it could be due to adaptation of cortical neural circuits that represent the filled-in square surface (the 'surface-adaptation hypothesis').

Shimojo and colleagues set out to discover which of these hypotheses was true. They used varying stimuli designed to induce different degrees of filling-in and different strengths of local afterimage, and asked people to rate the intensity and duration of the

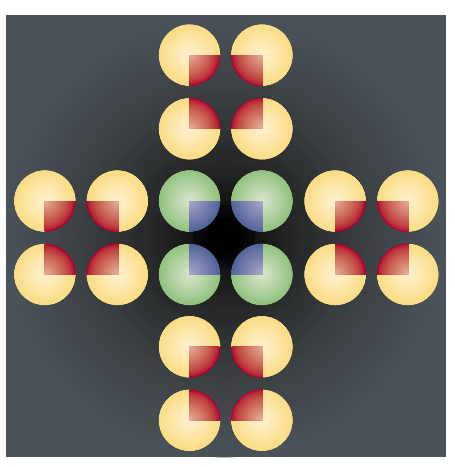

global afterimage. The results showed that the strength of the global afterimage did not depend on the strength of the local afterimages, but rather on the degree of filling-in that had been induced by the stimulus. This result was predicted by the surface-adaptation hypothesis, but would not have been expected if the global afterimage arose from the local afterimages.

Another unexpected finding was that the local and global afterimages appeared to rival each other, with most subjects reporting that they were seen separately but not together. This argues against even a compromise explanation, in which both proposed mechanisms contribute to the global afterimage. But the authors also report that some peripheral adaptation seems to be necessary for the global afterimage to be perceived - when only one eye was adapted, for example, the global afterimage was not seen by the unadapted eye.

Undoubtedly, there are still questions to be answered before we will fully understand the mechanisms of adaptation. But studies such as these will also help us to understand how the visual system represents surfaces, whether real or illusory.

Rachel Jones

\section{(2) References and links} ORIGINAL RESEARCH PAPER Shimojo, S. et al. Afterimage of perceptually filled-in surface. Science 293, 1677-1680 (2001)

FURTHER READING Pessoa, L. et al. Finding out about filling-in: a guide to perceptual completion for visual science and the philosophy of pertion for visual science and the philosophy of per-
ception. Behav. Brain Sci. 21, 723-748 (1998) | Wilson, H. R. A neural model of foveal light adaptation and afterimage formation. Vis. Neurosci. 14, 403-423 (1997)

WEB SITE

Shimojo's lab: http://neuro.caltech.edu/

\section{$\mathrm{A} \beta, \tau$ and the fA $\beta$ rica $\tau$ ion of $\tau$ angles}

A key question in the pathogenesis of Alzheimer's disease is the relation between the accumulation of $\beta$-amyloid $(A \beta)$ and the formation of neurofibrillary tangles. Are the two processes entwined or are they independent manifestations of the disease? Two recent papers provide convincing evidence that the two archetypal neuropathological changes seen in Alzheimer's disease are linked. Both papers converge on the idea that the formation of $\mathrm{A} \beta$ deposits acts to promote the appearance of tangles.

Lewis et al. generated a long-awaited transgenic animal - a mouse carrying mutant forms of both the amyloid precursor protein (APP) and tau, the main component of neurofibrillary tangles. Mice bearing either one of the two mutant genes fail to recapitulate all of the neuropathological changes found in Alzheimer's disease. It was therefore hoped that the doubletransgenic mice would constitute a better model of the pathology. Indeed, these mice showed both amyloid plaques and tangles but, more importantly, the number of tangles in the double mutant was higher than in mice expressing only tau, indicating that the accumulation of $A \beta$ might stimulate the formation of tangles.

But is $A \beta$ responsible for the observed phenotype or is it APP itself? In an independent study, Götz et al. used transgenic mice expressing tau but, instead of generating a double mutant, they injected $A \beta$ directly into the cortex and hippocampus of these animals. They found that this treatment also increased the number of tangles, providing evidence that $A \beta$ is indeed the molecule that stimulates tangle formation.

An intriguing aspect of both studies is that $A \beta$ and tau were spatially segregated. Lewis et al. found that, in contrast to what is found in Alzheimer's disease, amyloid plaques were not surrounded by tau-positive neurites in the double mutants, and Götz et al. found that tangle formation was stimulated in brain regions far from the $A \beta$ injection sites. So, it seems that these models also fail to reproduce every aspect of Alzheimer's disease, although the two of them provide better tools to test more rigorously the benefit of new treatments for this pathology. Juan Carlos López

\section{(D) References and links}

ORIGINAL RESEARCH PAPERS Lewis, $J$ et al. Enhanced neurofibrillary degeneration in transgenic mice expressing mutant tau and APP. Science 293, 1487-1491 (2001) | Götz, J. et al. Formation of neurofibrillary tangles in $\mathrm{P} 301 \mathrm{~L}$ tau transgenic mice induced by $\mathrm{A} \beta 42$ fibrils. Science 293, 1487-1491 (2001)

FURTHER READING Duff, K. \& Rao, M. V. Progress in the modeling of neurodegenerative diseases in transgenic mice. Curr. Opin. Neurol. 14, 441-447 (2001)

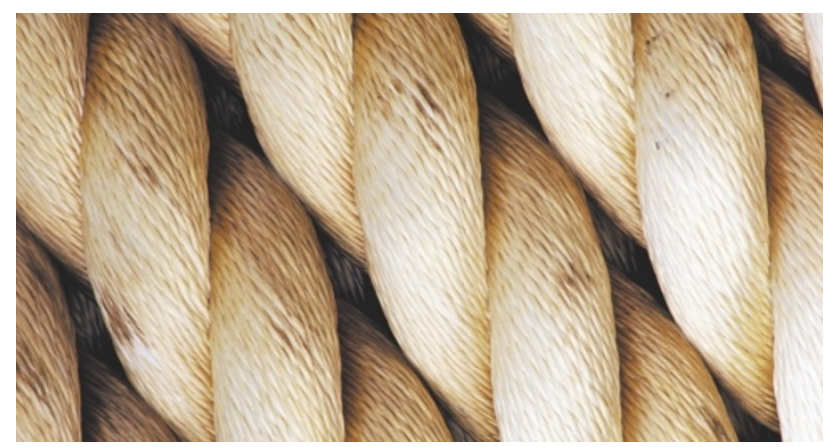

